\title{
Ian Snook Additional Prize 2016
}

The 2016 Ian Snook Additional Prize has been won by Diego Tapias, Alessandro Bravetti, and David Sanders. Their winning paper is published in this volume of the CMST [1]. We congratulate the Winners.

[1] D. Tapias, A. Bravetti, D.P. Sanders, Ergodicity of One-dimensional Systems Coupled to the Logistic Thermostat, CMST 23(1), 11-18 (2017).

Maciej Stroiński, Jan Węglarz, and Krzysztof Witold Wojciechowski CMST Editors, Poznań, Poland 